\title{
How can Clinical Research Trials Conducted in Developing Countries Minimize Exploitation?
}

\section{Mukherjee G*}

Department of Ethics, Vidyasagar University, India

*Corresponding author: Gia Mukherjee, Vidyasagar University, Rangamati, Midnapore, West Bengal, India, Email: gmukherjee624@yahoo.com

\section{Review Article \\ Volume 4 Issue 3}

Received Date: July 12, 2021

Published Date: August 06, 2021

DOI: $10.23880 /$ abca-16000191

\section{Abstract}

Western scholars, pharmaceutical companies, and academic institutions are and have been conducting research in developing countries for many years. These locations boast substantial cost savings, expedited timelines, and little to no regulatory oversight, making them attractive to developed-world researchers. Residents of these communities are impoverished, often illiterate, unemployed, and with untreated health conditions. Because established ethical protocols for international research are often compromised or misconstrued by researchers and/or their sponsors, these individuals are left vulnerable to exploitation and abuse.

In this paper, I explore how current utilization of ethical guidelines is enabling exploitation. I identify ethical questions regarding subject recruitment, informed consent, standard of care, and the post-trial obligations of researchers. I then examine specific situations in which exploitation occurred because of drug, vaccine, or clinical trials. I conclude by offering recommendations to create a more streamlined approach to international research that takes into account the experiences and needs of vulnerable populations. This approach helps ensure that participants are fully involved in the ethical approval process; able to choose whether or not to participate without any undue influence or pressure; treated with the standard of care best suited to their context and surroundings; and reasonably given access to any interventions proven effective during the course of the trial.. A respect for beneficence, justice, and self-autonomy should guide researchers' interactions with subjects before, during, and after the trial.

Keywords: Institutional Review Boards' (IRBs); National Institutes of Health; Gross Domestic Product; Methyl Isocyanate

\section{Aims}

- Explore the question of whether current research conduct fails to uphold established ethical principles and, as a result, enables exploitation.

- Examine the conditions under and implications of specific situations where the exploitation of vulnerable populations occurred because of drug, vaccine, or clinical trials.

- Offer recommendations for the ethical conduct of research in developing countries in regards to subject recruitment, informed consent, standard of care, and post-trial provision of resources.

\section{Background}

Human Subjects Research conducted by Western scholars, pharmaceutical companies, and academic institutions is and has been moving inexorably towards the developing world.

The increasingly bureaucratic, complex, and regulatory culture of research in wealthy countries places great burdens on investigators in terms of "compliance, documentation, 
and training" [1]. These hindrances can be easily overcome by outsourcing clinical trials to developing countries where, "the population size alone offers the promise of expanding markets" [2]. Trials in phases II and III can be easily conducted in these locations, "with the permission of the respective country's government and health authority" [3].

Researchers, specifically those working for pharmaceutical companies, can save a substantial amount of money by conducting research in emerging nations. One pharmaceutical executive reported that, "a first-rate academic medical center in India charges approximately $\$ 1,500$ to $\$ 2,000$ per case report; [This is] less than one tenth the cost at a second-tier center in the United States" (Sullivan, 2009). Since clinical research costs are driven by human labor, much of this cost difference is, "attributable to the lower salaries of physicians, nurses, and study coordinators" in emerging nations [4]. Because these professionals are often just as qualified as their developed world counterparts, investigators can significantly reduce trial expenditure while upholding the continued quality of their research. It is imperative that clinical trials address health challenges in a timely manner. However, it can take several years to process, review, and approve a clinical trial for usage in a developed country. This is due in part to the obstacles that investigators may face in securing adequate funding and support for their research.

For many pharmaceutical companies, this leaves only a small amount of time to market and benefit directly from their product. The diverse and easily accessible pool of potential research participants in developing countries provide opportunities to accelerate processes like subject recruitment, clinical testing, and data analysis. However, researchers' motivations for international research conduct often extend beyond its monetary value or its bureaucratic convenience. Developing countries also boast a relatively untapped potential for scientific conduct.

Patients in developing countries "often have untreated or undertreated diseases" (ASPE Editors) [4]. This gives researchers the opportunity to recruit patients who have not previously received medical treatment to their trial. For patients who are already receiving multiple effective therapies for a condition, it remains unclear whether adding a new agent would be beneficial, neutral, or detrimental, "on the basis of the findings of a successful placebo-controlled trial in a population of patients who have not previously received treatment" [5].

Research conducted in developing regions might also allow for the examination of diseases unique to those locations. Often, these types of investigations are not possible virtually or within the sponsoring nation, so it is necessary to outsource to a developing country.

\section{Challenges}

However, significant challenges may arise when conducting research in emerging nations. Wide disparities in educational levels, social structures, and healthcare systems make it difficult for researchers to protect the rights of vulnerable and disadvantaged populations in the host nation while also balancing the needs of wider society. These disparities put in jeopardy researchers' ability to provide a relevant informed consent that is appropriate to the culture of study and can adhere to global institutional review boards' (IRBs) pre-established ethical guidelines.

These shortcomings have significant implications for the livelihood and autonomy of the vulnerable individuals upon whom the research is being conducted.

\section{Vulnerability}

In the medical ethics literature, those identified as potentially vulnerable populations are those who are at risk of being taken advantage of during clinical research endeavors. In the Declaration of Helsinki, it is stated that: "Medical research with a vulnerable group is only justified if the research is responsive to the health needs or priorities of this group and the research cannot be carried out in a nonvulnerable group. In addition, this group should stand to benefit from the knowledge, practices or interventions that result from the research" (Declaration of Helsinki) [7].

Those generally accepted as being vulnerable include, "children, prisoners, pregnant women, fetuses, mentally disabled persons, and economically or educationally disadvantaged individuals" (Swarthmore Institutional Review Board, 2016). However, vulnerability is contextdependent and is not a static parameter in any way. Therefore, vulnerability in research occurs when a participant is incapable of protecting his or her interests and, as a result, has an increased probability of being intentionally or unintentionally exploited [8].

\section{Exploitation}

Another concern in medical ethics research is exploitation. As a general rule, it can be said that, "A exploits $B$ when $B$ receives an unfair level of benefits or unfair burden of risks as a result of interacting with A" [9]. Because A often benefits at B's expense, exploitation is thus understood as a kind of parasitism.

When researchers encounter individuals whose basic needs are unmet, they should help those individuals because 
of the inherent value they possess as a human being. Exploitation occurs when researchers see in the unmet basic needs of others an opportunity for profit rather than a cry for help [10]. Exploitation can, therefore, be defined as the use of populations [in] research from which they will not benefit [Belmont Report] [11]. Given this definition, it is necessary to ask whether the unfairness involved in exploitation confers any sort of harm to its victim and whether it is a violation of their moral rights.

Exploitation is often associated with the Kantian idea of respect for persons. A respect for others imposes upon us a duty of beneficence. It could be said that individuals are left vulnerable to exploitation and abuse when their basic needs are not met and they are being treated simply as a means to an end.

From a Marxian standpoint, however, exploitation is transaction specific and not necessarily dependent on the compliance or harming of the individual. Rather, it concerns the outcome of case-specific transactions, "allowing individuals to be exploited even when they consent to the transaction and [may even] benefit from it" [12]. Vulnerability, in this view, is neither necessary nor sufficient for exploitation.

The exploitation of subjects or host communities is minimized in developed countries because the researchers or research institutions are legally regulated by the larger community of which they are part. By facilitating interactions with regulatory bodies, they are able to translate research results into practices that benefit society directly.

To safeguard research participants abroad from harm or exploitation, several international guidelines ${ }^{1}$ recommend that externally sponsored research be reviewed by ethics committees or IRBs from both the sponsor and [the] host country [13]. However, local IRBs may lack the expertise or power to reject protocols approved by their developed country counterparts.

Moreover, regulatory infrastructures and, "independent oversight processes [aiming to minimize the risk of exploitation] might [also] be less well established, less supported financially, and less effective" in developing countries [14]. Factors like poverty, illiteracy, limited healthcare services, and cultural differences might exacerbate these differences.

Ultimately, this means that the responsibility to uphold ethical standards is often relegated to the researchers

1 These guidelines include, but are not limited to, the Belmont Report, the Declaration of Helsinki, and the Nuremberg Code. themselves who lack the incentives, capacity, or power to put individuals' wellbeing over the scientific and financial success of their trial.

\section{Ethical Questions}

There are several ethical questions that arise from research conduct in developing countries. These might include:

- Are subjects recruited in an ethically appropriate manner?

- Is adequate informed consent obtained?

- Is a sufficient standard of care maintained during the trial?

- Do subjects benefit from the research?

Although existing regulations and frameworks clearly highlight these principles, unethical conduct continues to occur. Current utilization of these protocols appears to be flawed because it has led to and further enabled situations involving the exploitation of vulnerable populations for scientific gain.

\section{Subject Recruitment}

In resource-limited settings, "healthy volunteers are most often poor people who might not understand the risks they are taking" by participating in research Bompart F, et al. [15]. They might, instead, see foreign researchers as well-meaning and knowledgeable figures of authority. This furthers the misconception that taking part in a clinical trial or any research proposed by a health care provider will benefit the patient population.

Historically, some researchers and research institutions have preyed on this misconception to advance their own clinical trials or scientific studies. In December 2000, a news article disclosed that a Chinese American researcher of a renowned American University had been, "collecting blood samples from villagers living in the Dabie Mountain region of China's Anhui province since 1995 with the financial support of the National Institutes of Health (NIH) and [other Western] bio pharmacy companies" [16]. It was alleged that the blood samples were then, "transferred to the US university's genetic bank for research into asthma, diabetes, hypertension" and other diseases [17]. Villagers were rewarded with a small monetary reward for their participation. Many of these participants hailed from the lowest rungs of society and were illiterate or unemployed.

This case poses a risk of exploitation because Anhui is "not as economically advanced as its neighboring provinces", so its citizens are generally less educated and more susceptible to unethical recruitment practices [16]. 
Per Capita Gross Domestic Product (GDP) in Northeast China in (Graph).

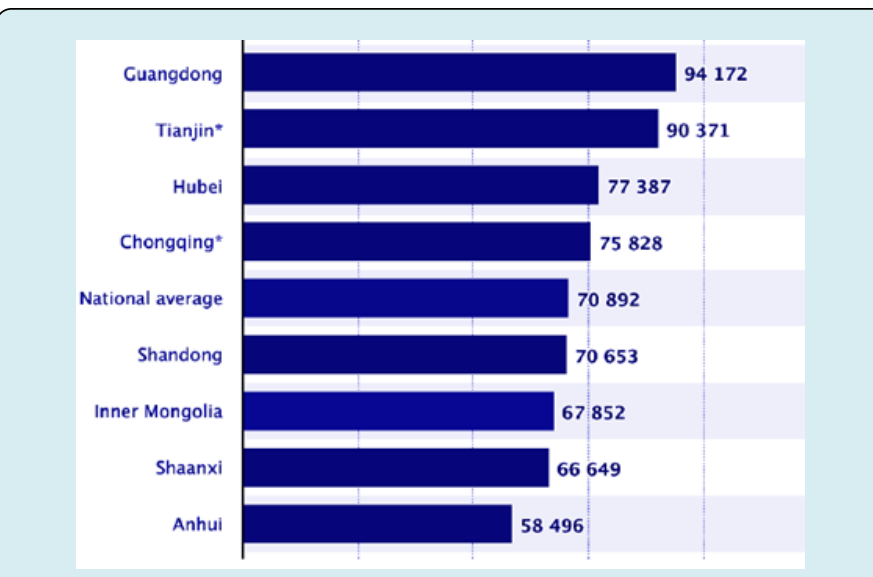

Graph: In 2019, the GDP per capita in Anhui was 58,496 yuan ( 9046 USD), far lower than those of the more developed neighboring provinces like Guangdong (94,172 yuan, 14557 USD) and Hubei (77387yuan, 11962 USD).

Generally, a region's economic bearings influence social factors like residents' education levels, incomes, and employment statuses. By conducting research in an area that is historically impoverished and underrepresented, foreign investigators can evade repercussions for ethical misconduct. Examples of misconduct include overly emphasizing compensation benefits, claiming that participation will improve subjects' health, and falsely advertising free medical treatment and care. These practices are seen as exploitative and grossly misconstrue the protocols established by international IRBs.

\section{Informed Consent}

Obtaining informed consent is essential to ethical conduct of clinical research. The Belmont Report defines informed consent as: The "respect for persons" requires that subjects, to the degree that they are capable, be given the opportunity to choose what shall or shall not happen to them (Belmont Report) [11].

However, many researchers fail to recognize and uphold these standards. A prime example of this failure is the Bhopal Gas Tragedy. This was a gas leak that occurred at the Union Carbide India Limited pesticide plant in Bhopal, India in 1984. Over 500,000 people were exposed to toxic methyl isocyanate (MIC) gas as a result of the leak [18]. Between 2004 and 2008, pharmaceutical companies like AstraZeneca, GlaxoSmithKline, and Pfizer conducted ten different clinical trials on the 215 survivors of the tragedy. They were testing the drugs Fondaparinux, Tigecycline, and Televancin.
Trial participants were illiterate and belonged to disadvantaged areas of society. Many alleged that they had signed up, "believing that they were getting vaccinated" and that they, "were not informed about the risks involved despite asking" [19]. One participant recalled, "Had I known it was for a trial, I would have never taken the jab" [20].

In the developed world, informed consent procedures, "rely primarily on individual autonomy, written documents requiring signatures, patients' prior experience with western medicine, and legal disclaimers to limit liability" [21]. However, evidence seems to indicate that these practices are not adequately utilized in developing countries.

Low rates of literacy and eminent language barriers may complicate the usage of established research methods with foreign subjects. Individuals may not understand the nature, objectives, and goals of the study. Rather than adapting to and finding creative solutions for these problems, researchers have, historically, forced or duped participants into cooperating.

The consent process cannot be transferred from the developed to developing world without considering the cultural, socioeconomic, and educational factors that influence international research. A failure to realize and acknowledge these factors limits the widespread implementation and usage of existing ethical models and frameworks.

\section{Standard of Care}

In recent years there has been intense debate regarding "the level of medical care provided to control groups in clinical trials conducted abroad" [22]. The debate revolves around the issue of how to define a standard of medical care in a country in which, "many people are not receiving the best methods of medical care available in other settings" [22].

The National Institute of Health (NIH) defines the standard of care as "a treatment that experts agree is appropriate, accepted, and widely used [NIH Editors] [23]. It is further defined by Dawson et al, as "the level of treatment that ought to be delivered under conditions of appropriate and efficient referral in a national system" [22].

Given this definition, it is evident that what constitutes a standard of care in a developed country might be difficult to identify and follow in a developing country. The question then becomes whether research that does not employ a universal standard of treatment should be considered acceptable. By providing, "participants [who are already in a vulnerable situation] because of their illness with a sub-standard intervention, researchers expose individuals 
and their communities to unnecessary risks" [24]. When populations are not provided with the best proven medicine, researchers are deviating from their duty to protect and act in the best interests of their subjects.

Three clinical trials that took place in rural areas of Mumbai, Osmanabad, and Dindigul [India] from 1998 to 2015 have faced heavy criticism for this infraction. The trials aimed to determine, "whether researchers could conduct cervical cancer screening using cheap methods of testing primarily visual inspection with acetic acid to reduce the incidence and mortality rate of cervical cancer" [25]. The trials were conducted on "approximately 374,000 women, [of whom] about 141,000 were placed in the control arm" [26]. Although the standard of care for testing of cervical cancer in India, "has been cytology screening since the 1970s, the technique was not available under governments universally" and for the purposes of the study was "misconstrued to be no screening" [26]. Thus, known and effective methods of screening for cervical cancer were deliberately withheld from over 141,000 women in areas where cervical cancer was known to be of high incidence and prevalence. Many participants' cancers were not detected and treated in time. As per the latest published reports on the incident, 254 women in the no-screening arm have died due to cervical cancer.

This case, along with many others of similar nature and methodology, are well documented in the medical ethics literature. However, it seems apparent that researchers are failing to implement the lessons learnt from these cases into their own trials. This furthers the cycle of exploitation and abuse in the international research setting.

\section{Post-Trial Provision}

It could be said that the failure to provide medical benefits to research subjects sacrifices their well-being for the sake of scientific knowledge. This is a violation of participants' moral right to not to be used as a means to a scientific end.

Reasoned availability, a phrase many authors refer to in the medical ethics literature, describes the idea that the products, "shown to be effective during the course of trial should be made reasonably available to the [trial] participants" and host community [14]. The Declaration of Helsinki describes this process as such:

"In advance of a clinical trial, sponsors, researchers and host country governments should make provisions for posttrial access for all participants who still need an intervention identified as beneficial in the trial" (Declaration of Helsinki) [7].
This approach has been implemented in many HIV trials, although generally for a specified period of time only [27]. For example, "13 out of 18 NIH-funded HIV antiretroviral therapy trials conducted during 2005-2007 in [developing countries] included post-trial access for trial participants", although long-term access to the antiretroviral therapy was not guaranteed [28].

Because participants are in great need, researchers might feel an emotional obligation to provide them with resources proven effective during the trial. These obligations might also stem from a desire to rectify past "historical injustices imposed on people from developing countries" or to relieve participants of any difficulties that they might have endured during the course of the trial [29].

However, most clinical trials fail to identify a safe and effective middle ground for collaboration with the trial participants and/or host country. Current utilization of this requirement is flawed because most researchers do not elucidate to participants whether or not they will receive sufficient compensation for their participation in the trial after it is over.

\section{Addressing the Challenges}

\section{Subject Recruitment}

There are a number of challenges that plague subject recruitment in international research. However, steps can be taken to mitigate the damage done to individuals and their communities.

Participation in research must, above all, be voluntary. To avoid coercion or undue influence, the recruitment process should provide participants with the tools they need to decide whether or not they wish to take part in the trial. In addition, sufficient time should be allocated to, "communicate a thorough explanation of the study" to participants [30]. Researchers should not try to pressure or intimidate participants in any way.

Often, participants are attracted to foreign research studies because of the health, economic, and social benefits they advertise. However, investigators should ensure that the information they present is "balanced" and "free of any misleading emphasis" that makes the study excessively attractive (Iowa State University Institutional Review Board) [31]. "They should not emphasize or overstate any anticipated benefits of the research".

Patients often think that "taking part in a clinical trial or any research proposed by a health care provider will benefit them" (Iowa State University Institutional Review Board, 
2016) [31]. "The recruitment methods and materials should avoid contributing to this misconception by using careful wording (ie:"using "research subject" instead of "patient," avoiding the term "treatment", etc") when advertising the trial [Iowa State University Institutional Review Board] [31]."

\section{Informed Consent}

The recruitment process of participants in developing countries is often undermined by a "poorly communicated" and hence "poorly understood" informed consent [27]. Although methods of obtaining sufficient consent differ on a case-by-case basis, general guidelines can be established.

Illiteracy is a substantial problem in international research. Researchers must develop strategies to eliminate the use of written documents when working with illiterate participants. They can present important documents containing information about the nature and objectives of the study to illiterate participants via a spoken phone or video message in their native language. If electronics are not available, a translator should be utilized.

Information about the nature and objectives of the trial should be clearly presented. It should fully align with the proposed research plan, be easily understandable, and, "be free from any technical or scientific jargon" (Iowa State University Institutional Review Board) [31].

When working with groups who are literate but cannot understand the proposed subject matter, an interactive approach is necessitated. Researchers should develop strategies to assess a potential participant's understanding of the protocol before they are allowed to enroll or participate in the trial. Formal checks of participants' understanding should be utilized, structured, for instance, into a quiz or questionnaire. Participants should have the opportunity to ask questions about anything that they do not understand, take the consent document home to discuss with their family, friends, or advocates, and be given enough time to decide whether or not to participate in the trial. Furthermore, avenues for ongoing communication and dialogue between the researcher and participation should remain open during and after the data collection process.

Researchers must also adapt to participants' social or cultural beliefs when seeking their consents. Beyond simply being asked for informed consent, community members should be "trained and eventually involved in the ethical approval process" (Iowa State University Institutional Review Board) [31]. They should be made aware of the limits of confidentiality and "any duties the researchers may have to report certain findings" [NAP] [20].
When appropriate, there is a need to engage community leaders in the consent process to ensure that participants understand what participation entails, its potential risks, and its potential benefits when providing consent. Often individuals will have formed a certain level of rapport with these leaders beforehand, so they are more likely to engage and cooperate with researchers if a trusted figure of authority does the same.

Researchers might encounter situations where gender and societal power structures come into play. When a woman's husband appears to be making decisions for her, researchers should contact the woman in isolation to make sure that she wants to participate in the trial. They should also give her an "out" so that she doesn't have to face the consequences of not participating in the trial. An alternate point of approach might be to contact the man and make sure that he understands his Wife's rights.

\section{Standard of Care}

The Declaration of Helsinki endorses the view that all trial participants in every country are entitled to the worldwide best standard of care [Declaration of Helsinki] [7]. However, a "blanket" definition of standard of care cannot be reasonably applied to the context of developing nations. What is considered a standard of care in the developed world could either be "irrelevant to the needs of research subjects" in the host country or not necessarily be "the best way to spend trial resources" in the interests of wider society (BMJ Editors) [32]. The standard of care should be specifically tailored to fit the context and needs of the patient population.

Researchers should aim to provide the subjects with a level of care and treatment that they would not ordinarily get in their home country. In doing so, they must make sure that existing disparities are not more deeply entrenched by the, "inappropriate deflection of local human or material resources away from the healthcare system in the host country" towards the research project [32]. All members of the research team should have equivalent qualifications, training, and expertise so that some participants do not receive more care and attention than others.

It should also be ensured that international researchers understand and are sensitive to the social, economic, and political context framing their research. They can facilitate cooperation by involving "members of the host country in the design and conduct of the trial" [24]. Researchers and their sponsors should be educated about research ethics and, more specifically, exploitation in research settings.

It will not be possible to achieve these standards merely by, "changing research declarations or by advancing the 
simplistic notion that ethical behavior can be promoted by such declarations" [33]. Rather, researchers must set and adhere to a broader moral agenda. To achieve this, they should consider the ways in which their actions contribute to the widening disparities in human wellbeing between developing and developed world settings. The highest achievable standard of care should be the goal, though "reasonable limits can be negotiated in specific contexts" [32]. However, the objective should be to ratchet the standard upward rather than to set utopian benchmarks that cannot be met.

\section{Post-Trial Access}

Although several possibilities have been suggested, there is discord as to what exactly justifies post trial obligations and who is responsible for them. The extent to which these obligations are affected by other considerations such as, the "scarcity of resources in the host country, the risk benefit ratio of the study, and the depth and length of the investigatorparticipant relationship" largely remains undetermined [27].

Post-trial access is usually determined on a case-by-case basis. The post-trial provisions and responsibilities of the researchers should be clearly laid out for the local community in the form of an agreement or contract. This will help avoid any future occurrences of exploitation because participants will know "exactly what to do or expect once the trial is over" ((MRCT Center Post-Trial Responsibilities Framework) [34]. However, some general recommendations can be made to improve current utilization of ethical protocols.

Researchers should, above all, show beneficence towards participants after the trial is over. By participating in the trial, participants have willingly volunteered to help advance science. For this, researchers are obligated to offer them access to any beneficial intervention that is developed and proven effective during the course of the trial. If participants are put at risk at any point during the research, the failure or refusal to provide them with these provisions is considered by many to be unfair and exploitative.

The burdens and benefits accrued as a result of the research should be distributed equally. Studies should not be conducted on vulnerable populations only for the benefits to be enjoyed solely by other, more well-off communities. To mitigate this possibility, sponsors should, "seek joint initiatives of international agencies and through privatepublic partnerships to produce affordable drugs" and other beneficial interventions [3].

In doing so, they must take "great care in assuring that the community and/or participants are not left 'worse off' than they...were before beginning the trial" [27]. After the research has concluded, "sponsors should help strengthen health-delivery systems" in the region and focus on capacity building. They should also train personnel specifically to transfer the skills learned in a way that empowers participants to take charge after researchers have left [3].

\section{Limitations}

Exploitation is prone to occur in the developing world setting regardless of the regulations, guidelines, and protocols that researchers put in place. The financial, bureaucratic, and scientific incentives to conduct research in developing countries are cyclical and, realistically, are unlikely to become less influential in the future.

It is to be noted that the potential scope, magnitude, and effectiveness of these recommendations are limited [35,36]. Most researchers will take them at face value, misconstruing them as they do the existing ethical protocols.

There is, at present, a need to clarify the ways in and methods through which researchers can modify their own practice and pedagogy to fit an ethically acceptable model for international research. Compliance with the above recommendations will help create a research environment of safety, trust, and communication while also limiting the likelihood for the exploitation or mistreatment of trial participants [37]. Ultimately, it is up to the researchers themselves to weigh the ethical benefits and drawbacks of outsourcing their trial to a developing country [38].

\section{Conclusion}

Researchers are incentivized to conduct research abroad because of low costs, shortened timelines, and scientific potential. The historical cases presented make apparent that existing ethical protocols are not being utilized effectively and in an appropriate manner by researchers, their sponsors, and their institutions. The established recruitment, consent, care, and post-trial processes are often compromised, leaving subjects vulnerable to exploitation and abuse $[39,40]$. To mitigate this possibility and elucidate the research process for all parties involved, it should be ensured that subjects are informed about the nature, objectives, and methods of the trial; can choose whether or not to participate freely and without undue influence or pressure; are treated with the standard of care best suited to their context and surroundings; and are reasonably given access to the interventions proven effective during the course of the trial [39].

Although researchers' obligations to their subjects will vary on a case-by-case basis, a respect for beneficence, justice, and self-autonomy should guide their interactions with subjects before, during, and after the trial. 


\section{References}

1. Glickman SW, John GM, Eric DP, Charles BC, Robert AH, et al. (2009) Ethical and Scientific Implications of the Globalization of Clinical Research. The New England Journal of Medicine 360(8): 816-823.

2. Ruiz MA (2012) Challenges of emerging countries: a relatively positive view for the future Europe PMC 34(3): 173-174.

3. Soni U, Singh M (2013) Clinical Trials Outsourcing: Good or Bad?. Drug Designing Open Access 2(1): 1000104.

4. Lang T, Siribaddana S (2012) Clinical Trials Have Gone Global: Is This a Good Thing? PLoS Medicine 9(6): e1001228.

5. (2014) Barriers Related to the Globalization of Clinical Research. ASPE.

6. Usha G, Menka V (2013) Placebo in Clinical Trials. Perspectives in Clinical Research 4(1): 49-52.

7. (2021) WMA Declaration of Helsinki-Ethical Principles for Medical Research Involving Human Subjects. World Medical Association.

8. Alejandra GD, Elena ZG, Heriberto MF, Josefina AG, Marta DC et al. (2019) II. The Research Ethics Involving Vulnerable Groups. Rev Invest Clin 71(4): 217-225.

9. Denny CC, Christine G (2007) Clinical Research with Economically Disadvantaged Populations. Journal of Medical Ethics 33(7): 382-385.

10. Zwolinski M, Wertheimer A (2017) "Exploitation". The Stanford Encyclopedia of Philosophy. In: Edward N, et al. (Eds.), Metaphysics Research Lab, Stanford Encyclopedia of Philosophy.

11. (2019) Read the Belmont Report.

12. Rafael DR, Annette R, Ezekiel E, David W (2016) The Potential Exploitation of Research Participants in High Income Countries Who Lack Access to Health Care. British Journal of Clinical Pharmacology 81(5): 857-864.

13. Chuan VT, Schaefer GO (2015) Research in ResourcePoor Countries. The Hastings Center.

14. Emanuel EJ, David W, Jack K, Christine G (2004) What Makes Clinical Research in Developing Countries Ethical? The Benchmarks of Ethical Research. The Journal of Infectious Diseases 189(5): 930-937.

15. Bompart F (2019) Healthy Volunteers for Clinical
Trials in Resource-Poor Settings: National Registries Can Address Ethical and Safety Concerns. Cambridge Quarterly of Healthcare Ethics 28(1): 134-143.

16. Zvonimir K (2018) Ethics and Integrity in Health and Life Sciences Research. Emerald Group Publishing 4: 239.

17. Yandong Z, Zhang W (2018) An International Collaborative Genetic Research Project Conducted in China. Ethics Dumping: Case Studies from North-South Research Collaborations, Springer Link, pp: 71-80.

18. Mandavilli A (2018) The World's Worst Industrial Disaster Is Still Unfolding. The Atlantic.

19. (2021) No Consent, No Follow-up, Claim Bhopal Gas Tragedy Victims in Vaccine Trial. The Economic Times.

20. (2021) Read Ethical Considerations for Research on Housing-Related Health Hazards Involving Children. NAP.

21. Donald JK, Diop S, Diallo A, Mzayek F, Keating J, et al.(2010) Informed Consent in International Research: The Rationale for Different Approaches. The American Journal of Tropical Medicine and Hygiene 83(4): 743747.

22. Hyder AA, Liza D (2005) Defining Standard of Care in the Developing World: The Intersection of International Research Ethics and Health Systems Analysis. Developing World Bioethics 5(2): 142-152.

23. (2021) Per capita gross domestic product (GDP) of Anhui province, China from 2010 to 2019.

24. Lignou S (2011) The 'Standard of Care' Debate and Global Justice in Research. Research Ethics 7(1): 5-12.

25. Veena J (2017) Cervical Cancer Screening in India. Ethics Dumping: Case Studies from North-South Research Collaborations, pp: 1-144.

26. Sandhya S (2018) Cervical Cancer Screening in India. Ethics Dumping: Case Studies from North-South Research Collaborations. Springer, pp: 33-48.

27. Khan N (2014) The Post-Research Ethical Obligations of the Research Enterprise in Developing Countries. Journal of Clinical Research \& Bioethics 5(4): 1000194.

28. Shah S, Elmer S, Gradyet C (2009) Planning for Posttrial Access to Antiretroviral Treatment for Research Participants in Developing Countries. American Journal of Public Health 99(9): 1556-1562.

29. Shen L (2008) International Literacy Statistics: A Review 
of Concepts, Methodology and Current Data. UNESCO Institute for Statistics.

30. Wilson S, Draper H, Ives J (2008) Ethical Issues Regarding Recruitment to Research Studies within the Primary Care Consultation. Fam Pract 25(6): 456-461.

31. (2016) Recruitment of Research Participants. Iowa State University Institutional Review Board.

32. Solomon BR, Singer PA (2000) A New Look at International Research Ethics. BMJ: British Medical Journal 321(7264): 824-826.

33. Vivekanand RJ, Jicheng L, Ranjit RC, Yongjun W, et al. (2016) International Trials in Middle-Income Countries: Different Local Scenarios Require Different Ethical Approaches. Journal of the Royal Society of Medicine 109(2): 47-51.

34. (2017) MRCT Center Post-Trial Responsibilities Framework. The MRCT Center of Brigham and Women's Hospital and Harvard.
35. Nagarajan R (2014) Row over Clinical Trial as 254 Indian Women Die. The Times of India.

36. Diemert DJ, Lucas L, Ashley S, Maria Z, Amanda S et al. (2017) A Comparison of the Quality of Informed Consent for Clinical Trials of an Experimental Hookworm Vaccine Conducted in Developed and Developing Countries. PLoS Neglected Tropical Diseases 11(1): e0005327.

37. Sullivan T (2009) Health Care Reform: Clinical Trials Leaving the US. Policy \& Medicine.

38. (2012) Madhya Pradesh Government: Bhopal Gas Tragedy Relief and Rehabilitation Department, Bhopal.

39. Schroeder D, Cook J, Hirsch F, Fenet S, Muthuswamy V (2017) Ethics Dumping: Case Studies from North-South Research Collaborations.

40. Seema Shah JD, Stacey Elmer BA, Grady C (2009) Planning for Posttrial Access to Antiretroviral Treatment for Research Participants in Developing Countries. American Journal of Public Health 99(9): 1556-1562. 